\title{
Bernstein and Bernstein-Like Inequalities for Modulation Spaces
}

\author{
Sa'ud Al-Sa'di ${ }^{*}$ and Salti Samarah ${ }^{2}$ \\ ${ }^{1}$ Department of Mathematics, Faculty of Science, The Hashemite University, P.O Box 330127, Zarqa 13133, Jordan \\ ${ }^{2}$ Department of Mathematics and Statistics, Jordan University of Science and Technology, Irbid 22110, Jordan \\ * Corresponding author
}

\begin{abstract}
It is shown that the modulation spaces $M_{p}^{w}$ can be characterized by the approximation behavior of their elements using Local Fourier bases. In analogy to the Local Fourier bases, we show that the modulation spaces can also be characterized by the approximation behavior of their elements using Gabor frames. We derive direct and inverse approximation theorems that describe the best approximation by linear combinations of $\mathrm{N}$ terms of a given function using its modulates and translates.
\end{abstract}

Keywords: Nonlinear approximation; Modulation spaces; Gabor frames; Bernstein inequality.

2010 Mathematics Subject Classification: 41A17, 41A46; 46B15; 42 C15.

\section{Introduction}

One of the central problems of approximation theory is to characterize the set of functions which have a prescribed order of approximation by a given method of approximation, e.g., to characterize the functions which have approximation order $\mathscr{O}\left(N^{-\alpha}\right)$ for some fixed $\alpha>0$. Results of this type are known and easy to prove when the space to work in is a Hilbert space and the set where the approximation is seeking is an orthonormal system.

For general systems, there are sufficient conditions on a given function in the space which guarantees certain rate of decrease for the error of approximation. In this paper we consider the method of nonlinear approximation in particular spaces called the modulation spaces. We investigate the approximation of smooth functions by time-frequency shifts. The method of nonlinear approximation has recently found many computational applications such as data compression, statistical estimation or adaptive schemes for partial differential or integral equations.

Nonlinear approximation is utilized in many numerical algoritheorems, it occurs in several applications. In mathematics and applications it is very important to write a function in some function space in the form

$f=\sum_{k \in \Lambda} \lambda_{k} g_{k}$

where $\Lambda$ is an indexed set and $\left\{g_{k}: k \in \Lambda\right\}$ is a set of functions. The case in which this set is obtained from a single function is very interesting. One way to construct such set is by using the Gabor frames. Here we characterize functions with a given degree of nonlinear approximation by linear combination of $N$ terms of a given function $f$ belonging to some specific space using its modulates and translates.

Let $X$ be a Banach space, where the approximation takes place, with norm

$\|\|=.\|\cdot\|_{X}$. Then we say that a subset $\mathscr{D}$ of functions from $X$ is a dictionary if :

for all $g \in \mathscr{D},\|g\|_{X}=1$, and $g \in \mathscr{D}$ implies $-g \in \mathscr{D}$ 
Let $\Sigma_{N}(\mathscr{D})$ be the set in which the approximation is seeked, which denotes the collection of all functions in $X$ which can be written as a linear combination of at most $N$ elements of $\mathscr{D}$, i.e.,

$\Sigma_{N}(\mathscr{D})=\left\{s \in X ; s=\sum_{k \in F} c_{k} g_{k}, g_{k} \in \mathscr{D}, c_{k} \in \mathbb{C}, \operatorname{card}(F) \leq N\right\}$

From this definition we note that the sum of two elements from $\Sigma_{N}$ is generally not in $\Sigma_{N}$, and needs $2 N$ terms in its representation by the $g_{k}$ 's, so it is rather to be in the larger set $\Sigma_{2 N}$, which means that the space $\Sigma_{N}$ is not linear, for this reason best $N$-term approximation is often called nonlinear approximation.

For any given $f \in X$, the error associated to the best $N$-term approximation to $f$ from $\mathscr{D}$ is given by:

$\sigma_{N}(f, \mathscr{D})_{X}:=\sigma_{N}(f)_{X}=\inf _{s \in \Sigma_{N}}\|f-s\|_{X}$

The method of nonlinear approximation was first used by Stechkin; he characterized the space of all $f \in L_{2}(\mathbb{R})$ which have absolutely convergent orthogonal expansion, see [1]. More precisely, he proved the following theorem:

Theorem 1.1. ([2]). Let $\left\{\phi_{k} ; k \in \mathbb{N}\right\}$ be an orthonormal basis for $L_{2}(\mathbb{R})$. Then for $f \in L_{2}(\mathbb{R})$ we have:

$\sum_{k \in \mathbb{N}}\left|\left\langle f, \phi_{k}\right\rangle\right|<\infty \Longleftrightarrow \sum_{N=1}^{\infty}\left(N^{\frac{1}{2}} \sigma_{N}(f)\right) \frac{1}{N}<\infty$

Moreover, if the orthonormal basis is the trigonometric basis $\left\{e^{2 \pi i k x} ; k \in \mathbb{Z}\right\}$, and if $\bar{T}$ denotes the torus (like the interval $[0, \pi]$ ), then we have the following result.

Corollary 1.2. ([2]). Let $\mathscr{A}(\bar{T})$ be the space of all $f \in L^{2}(\bar{T})$ which have an absolutely convergent Fourier Series. Then for $f \in L^{2}(\bar{T})$

$f \in \mathscr{A}(\bar{T}) \Longleftrightarrow \sum_{N=1}^{\infty}\left(N^{\frac{1}{2}} \sigma_{N}(f)\right) \frac{1}{N}<\infty$

For a general discussion of the characterization problem, DeVore and Temlyakove made a modification of Stechkin's result to characterize other approximation spaces: For a general dictionary $\mathscr{D}$, and for any $p>0$ define

$A_{p}^{o}(\mathscr{D}, M)=\left\{f \in \mathscr{H} ; f=\sum_{k \in \Lambda} c_{k} w_{k}, w_{k} \in \mathscr{D},|\Lambda|<\infty\right.$ and $\left.\left(\sum_{k \in \Lambda}\left|c_{k}\right|^{p}\right)^{\frac{1}{p}} \leq M\right\}$

and we define $A_{p}(\mathscr{D}, M)$ as the closure (in $\mathscr{H}$ ) of $A_{p}^{o}(\mathscr{D}, M)$. Furthermore, define

$A_{p}(\mathscr{D})=\bigcup_{M>0} A_{p}(\mathscr{D}, M)$

and for $f \in A_{p}(\mathscr{D})$ the semi-norm $|f|_{A_{p}(\mathscr{D})}$ is the smallest $M$ such that $f \in A_{p}(\mathscr{D}, M)$.

Let $\mathscr{D}$ be given by an orthonormal basis $\left\{\phi_{k} ; k \in \mathbb{Z}\right\}$. Then $f \in A_{p}(\mathscr{D})$ if and only if $\sum_{k}\left|\left\langle f, \phi_{k}\right\rangle\right|^{p}<\infty$ and

$|f|_{A_{p}(\mathscr{D})}=\left(\sum_{k \in \mathbb{Z}}\left|\left\langle f, \phi_{k}\right\rangle\right|^{p}\right)^{1 / p}$.

It means that we can characterize certain approximation orders by the spaces $\mathscr{A}_{p}$. So, Stechkin's result in Theorem (1.1) can be formulated as follows:

$f \in \mathscr{A}_{1}(\mathscr{D})$ if and only if $\sum_{N=1}^{\infty}\left(N^{1 / 2} \sigma_{N}(f, \mathscr{D})\right) \frac{1}{N}<\infty$.

A slight modification of Stechkin's result due to DeVore and Temlyakov is given in the following theorem.

Theorem 1.3. ([2]). If $\mathscr{D}$ is given by an orthonormal basis for $\mathscr{H}$, for $\alpha>0$ and $p=\left(\alpha+\frac{1}{2}\right)^{-1}$, we have :

$f \in \mathscr{A}_{p}(\mathscr{D}) \Longleftrightarrow \sum_{N=1}^{\infty}\left(N^{\alpha} \sigma_{N}(f)\right)^{p} \frac{1}{N}<\infty$ 
This theorem provides a characterization of functions with an approximate order like $\mathscr{O}\left(N^{-\alpha}\right)$, for $\alpha=\frac{1}{p}-\frac{1}{2}$, using an orthonormal basis, and as a special case, setting $p=1$ we get Stechkin's result. We are now interested in characterization the approximation space $\mathscr{A}_{p}(\mathscr{D})$ in the whole range of the parameters $\alpha, p$ and a given dictionary $\mathscr{D}$.

\section{Gabor Frames and Modulation Spaces}

In this section we will collects the necessary background on the Gabor frames and modulation spaces. The modulation spaces occur in the study of the concentration of a function in the time-frequency plane. They were introduced in 1983 by $\mathrm{H}$. Feichtinger [3] and were subsequently investigated in [4, 5]. These spaces are defined by the decay properties of the short time Fourier transform, and contain many classical function spaces.

In addition to the basic definitions and notations of [6], the Schwartz class and the space of tempered distributions on $\mathbb{R}$ are denoted by $\mathscr{S}(\mathbb{R})$ and $\mathscr{S}^{\prime}(\mathbb{R})$ respectively. The translation and modulation of a function $f$ with domain $\mathbb{R}$ are defined, respectively, by:

$\boldsymbol{T}_{x} f(t)=f(t-x) \quad$ and $\quad \boldsymbol{M}_{w} f(t)=e^{2 \pi i w t} f(t)$

for $x, w \in \mathbb{R}$, where $\boldsymbol{T}_{x}$ is a translation by $x$ or time-shift, and $\boldsymbol{M}_{w}$ is a modulation by $w$ or frequency-shift. The Short-Time Fourier transform (STFT) of a function $f \in L^{2}(\mathbb{R})$ with respect to a function $g \in L^{2}(\mathbb{R})$ called the window function, is defined as:

$\mathscr{V}_{g} f(x, w)=\int_{\mathbb{R}} f(t) \bar{g}(t-x) e^{-2 \pi i w t} d t=\left\langle f, \boldsymbol{M}_{w} \boldsymbol{T}_{x} g\right\rangle$.

For any continuous strictly positive function $m$ on $\mathbb{R}$, the weighted $L_{p}$ space $L_{p}^{m}(\mathbb{R})$ is defined by the norm $\|f\|_{L_{p}^{m}}=\|f m\|_{L_{p}}$

and the mixed-norm spaces $L_{p, q}^{m}\left(\mathbb{R}^{2}\right)$ consists of all (Lebesgue) measurable functions on $\mathbb{R}^{2}$, such that for a weight function $m$ on $\mathbb{R}^{2}$ the norm

$$
\|F\|_{L_{p, q}^{m}}=\left(\int_{\mathbb{R}}\left(\int_{\mathbb{R}}|F(x, w)|^{p} m(x, w)^{p} d x\right)^{q / p} d w\right)^{1 / q}
$$

is finite. Throughout this paper, we will use two types of weights:

A submultiplicative weight function $v$ on $\mathbb{R}^{2}$ which is a positive, symmetric and continuous function and satisfies

$v\left(z_{1}+z_{2}\right) \leq v\left(z_{1}\right) v\left(z_{2}\right), \quad$ for all $z_{1}, z_{2} \in \mathbb{R}^{2}$.

An $v$-moderate weight function $m$ on $\mathbb{R}^{2}$ which is a positive, symmetric and continuous function and satisfies

$m\left(z_{1}+z_{2}\right) \leq C v\left(z_{1}\right) m\left(z_{2}\right), \quad$ for all $z_{1}, z_{2} \in \mathbb{R}^{2}$.

Given a non-zero window function $g \in L_{2}(\mathbb{R})$ and constants $\alpha, \beta>0$, the set of time-frequency shifts

$\mathscr{G}(g, \alpha, \beta)=\left\{\mathbf{T}_{\alpha k} \mathbf{M}_{\beta n} g ; k, n \in \mathbb{Z}\right\}$

is called a Gabor frame for $L_{2}(\mathbb{R})$ if there exists constants $A, B>0$ (called frame bounds) such that for all $f \in L_{2}(\mathbb{R})$

$A\|f\|_{L_{2}}^{2} \leq \sum_{k, n \in \mathbb{Z}}\left|\left\langle f, \mathbf{T}_{\alpha k} \mathbf{M}_{\beta n} g\right\rangle\right|^{2} \leq B\|f\|_{L_{2}}^{2}$

Proposition 2.1. ([6]). If $\mathscr{G}(g, \alpha, \beta)$ is frame for $L_{2}(\mathbb{R})$, then there exists a dual window $\gamma \in L_{2}(\mathbb{R})$, such that the dual frame of $\mathscr{G}(g, \alpha, \beta)$ is $\mathscr{G}(\gamma, \alpha, \beta)$. Consequently, every $f \in L_{2}(\mathbb{R})$ possesses the expansion:

$$
\begin{aligned}
f & =\sum \sum_{k, n \in \mathbb{Z}}\left\langle f, \mathbf{T}_{\alpha k} \mathbf{M}_{\beta n} g\right\rangle \mathbf{T}_{\alpha k} \mathbf{M}_{\beta n} \gamma \\
& =\sum \sum_{k, n \in \mathbb{Z}}\left\langle f, \mathbf{T}_{\alpha k} \mathbf{M}_{\beta n} \gamma\right\rangle \mathbf{T}_{\alpha k} \mathbf{M}_{\beta n} g .
\end{aligned}
$$


with unconditional convergence in $L_{2}(\mathbb{R})$. Further, the following norm equivalences hold:

$A\|f\|_{2}^{2} \leq \sum \sum_{k, n \in \mathbb{Z}}\left|\mathscr{V}_{g} f(\alpha k, \beta n)\right|^{2} \leq B\|f\|_{2}^{2}$

and

$B^{-1}\|f\|_{2}^{2} \leq \sum \sum_{k, n \in \mathbb{Z}}\left|\left\langle f, \mathbf{T}_{\alpha k} \mathbf{M}_{\beta n} \gamma\right\rangle\right|^{2} \leq A^{-1}\|f\|_{2}^{2}$.

for some constants $A, B>0$.

Under stronger assumptions on the function $g$, the expansion in equations (2.8) and (2.9) are valid not only in $L_{2}$ but in the entire class of function spaces, namely, the modulation spaces.

Definition 2.2. ([6]). Fix a non-zero window $g \in \mathscr{S}(\mathbb{R})$, a v-moderate weight function $m$ on $\mathbb{R}^{2}$, and $1 \leq p, q \leq \infty$. Then the modulation space $M_{p, q}^{m}(\mathbb{R})$ consists of all tempered distributions $f \in \mathscr{S}^{\prime}(\mathbb{R})$ such that

$\|f\|_{M_{p, q}^{m}}=\left\|\mathscr{V}_{g} f\right\|_{L_{p, q}^{m}}=\left(\int_{\mathbb{R}}\left(\int_{\mathbb{R}}\left|\mathscr{V}_{g} f(x, w)\right|^{p} m(x, w)^{p} d x\right)^{q / p} d w\right)^{1 / q}$

is finite.

Thus, $M_{p, q}^{m}$ is a Banach space of tempered distributions. If $p=q$, then we write $M_{p}^{m}$ instead of $M_{p, p}^{m}$, and if $m(z) \equiv 1$ on $\mathbb{R}^{2}$, then we write $M_{p, q}$ and $M_{p}$ for $M_{p, q}^{1}$ and $M_{p}^{1}$, respectively. Some examples of modulation spaces are the following:

1. The Segal algebra $S_{0}(\mathbb{R})=M_{1,1}(\mathbb{R})$.

2. $L_{2}(\mathbb{R})=M_{2,2}(\mathbb{R})$. However, $L_{p}$ does not coincide with any modulation space when $p \neq 2$ [7].

3. The Bessel potential space:

$$
H^{s}(\mathbb{R})=\left\{f \in \mathscr{S}^{\prime} ;\|f\|_{H^{s}}=\left(\int_{\mathbb{R}}|\hat{f}(w)|^{2}\left(1+|w|^{2}\right)^{2 s} d w\right)^{1 / 2}<\infty\right\} .
$$

Theorem 2.3. ([6]). Let $m$ be a v-moderate weight. Then

1. $M_{p, q}^{m}(\mathbb{R})$ is a Banach space for $1 \leq p, q \leq \infty$.

2. $M_{p, q}^{m}$ is invariant under time-frequency shifts, and $\left\|\mathbf{T}_{x} \mathbf{M}_{w} f\right\|_{M_{p, q}^{m}} \leq C v(x, w)\|f\|_{M_{p, q}^{m}}$.

3. If $1 \leq p_{1}, p_{2}, q_{1}, q_{2} \leq \infty$ and $m_{2} \leq C m_{1}$, then

$M_{p_{1}, q_{1}}^{m_{1}} \subseteq M_{p_{2}, q_{2}}^{m_{2}}$, whenever $p_{1} \leq p_{2}, q_{1} \leq q_{2}$.

Moreover, there exists a constant $C$ such that

$\|f\|_{M_{p_{2}, q_{2}}^{m_{2}}} \leq C\|f\|_{M_{p_{1}, q_{1}}^{m_{1}}}$

for all $f \in M_{p_{1}, q_{1}}^{m_{1}}$.

The appropriate window class in this setting is the Feichtinger algebra

$M_{1}^{v}=\left\{f \in \mathscr{S}^{\prime}(\mathbb{R}): \mathscr{V}_{f} f \in L_{1}^{v}\left(\mathbb{R}^{2}\right)\right\}$.

where $v$ is a submultiplicative weight on $\mathbb{R}^{2}$ with polynomial growth.

Theorem 2.4. ([6]). Suppose $1 \leq p, q \leq \infty$, $m$ is a v-moderate, $g, \gamma \in M_{1}^{v}$. Suppose that $\left\{\mathbf{M}_{\beta n} \mathbf{T}_{\alpha k} g, k, n \in\right.$ $\mathbb{Z}\}$ generates a frame for $L_{2}(\mathbb{R})$, then for all $f \in M_{p, q}^{m}$ we have

$$
\begin{aligned}
f & =\sum_{n \in \mathbb{Z}} \sum_{k \in \mathbb{Z}}\left\langle f, \mathbf{M}_{\beta n} \mathbf{T}_{\alpha k} g\right\rangle \mathbf{M}_{\beta n} \mathbf{T}_{\alpha k} \gamma \\
& =\sum_{n \in \mathbb{Z}} \sum_{k \in \mathbb{Z}}\left\langle f, \mathbf{M}_{\beta n} \mathbf{T}_{\alpha k} \gamma\right\rangle \mathbf{M}_{\beta n} \mathbf{T}_{\alpha k} g
\end{aligned}
$$

with unconditional convergence in $M_{p, q}^{m}$ if $p, q<\infty$, and weak-star convergence in $M_{\infty}^{1 / v}$ otherwise. Furthermore, there are constants $A, B>0$ such that for all $f \in M_{p, q}^{m}$

$A\|f\|_{M_{p, q}^{m}} \leq\left(\sum_{n \in \mathbb{Z}}\left(\sum_{k \in \mathbb{Z}}\left|\left\langle f, \mathbf{M}_{\beta n} \mathbf{T}_{\alpha k} g\right\rangle\right|^{p} m(\alpha k, \beta n)^{p}\right)^{q / p}\right)^{1 / q} \leq B\|f\|_{M_{p, q}^{m}}$ 
And the norm equivalence:

$A^{\prime}\|f\|_{M_{p, q}^{m}} \leq\left(\sum_{n}\left(\sum_{k}\left|\left\langle f, \mathbf{M}_{\beta n} \mathbf{T}_{\alpha k} \gamma\right\rangle\right|^{p} m(\alpha k, \beta n)^{p}\right)^{q / p}\right)^{1 / q} \leq B^{\prime}\|f\|_{M_{p, q}^{m}}$

holds on $M_{p, q}^{m}$.

\section{Characterization of Modulation Spaces $M_{p}$}

According to the definition of the approximation spaces $\mathscr{A}_{p}(\mathscr{D})$, several questions arise:

1. If $\mathscr{D}$ is given by the Fourier basis, what is the space that can be characterized by this basis?.

2. If $\mathscr{D}$ is given by a Local Fourier basis, what are the spaces that can be characterized by this basis?.

3. If $\mathscr{D}$ is given by Gabor frame, what are the spaces that can be characterized by this basis?.

The first question was answered by Stechkin in Theorem (1.1). For the second question we have the K. Gröchenig and S. Samarah result [8].

Theorem 3.1. ([8]). If $0<p<q \leq \infty$ and $\alpha=\frac{1}{p}-\frac{1}{q}$, then:

$f \in M_{p}^{w} \quad$ if and only if $\quad \sum_{N=1}^{\infty}\left(N^{\alpha} \sigma_{N}(f)_{M_{q}^{w}}\right)^{p} \frac{1}{N}<\infty$

In the proof of Theorem (3.1) the basis property was used in an essential way to rewrite the approximation error in terms of a sequence space norm. For linearly dependent sets it is not clear how much of Theorem (3.1) still holds. The next theorem prove the one-half of Theorem (3.1) under the weaker assumption that the set $\left\{\mathbf{T}_{\beta m} \mathbf{M}_{\gamma n} \phi: m, n \in \mathbb{Z}\right\}$ is a Banach frame for $M_{p}^{w}$.

Proposition 3.2. ([9]). Let $\left\{\mathbf{T}_{\beta m} \mathbf{M}_{\gamma n} \phi: m, n \in \mathbb{Z}\right\}$ be a Banach frame for $M_{p}^{\omega}$ for all $0<p<\infty$. If $0<p<q, \alpha=\frac{1}{p}-\frac{1}{q}$ and $f \in M_{p}^{\omega}$, then

$\sum_{N=1}^{\infty}\left(N^{\alpha} \sigma_{N}(f)_{M_{q}^{\omega}}\right)^{p} \frac{1}{N}<\infty$

Proposition (3.2) does not give a complete characterization of the modulation spaces $M_{p}$ using the Gabor atoms as a dictionary. It proves only that for a function $f$ in a modulation space $M_{p}$, the approximation error has order $N^{-\alpha}$, but does not give the other implication, i.e., if the approximation error for some function has order $N^{-\alpha}$ for some $\alpha>0$, what does this tell us about the space to which $f$ belongs. This inquiry will be answered in our next work which will based on the classical inequalities of Jackson and Bernstein where we applied with the Gabor atoms in the modulation spaces.

Let $X$ be a Banach space in which approximation takes place and assume that we can find a number $r>0$ and a second space $Y$ continuously embedded in $X$, and $X_{n}$ be the subsets of $X$ in which approximants come from. Then

Jackson Inequality: $\sigma_{n}(f)_{X} \leq C n^{-r}|f|_{Y}$, for all $f \in Y, n=1,2, \ldots$.

Bernstein Inequality: $|s|_{Y} \leq C^{\prime} n^{r}\|s\|_{X}$, for all $s \in X_{n}, n=1,2, \ldots \ldots$

Our claim now is: Assuming that the modulation space $M_{\infty}$ is the space in which approximation takes place, and using $\Sigma_{N}$ as the subset of $X$ in which the approximants are seeked. Then Bernstein inequality holds for our working space. Before proving our new result let we define $\Sigma_{N}$ using Gabor frames as follows:

$\Sigma_{N}(\mathscr{D})=\left\{s \in M_{\infty} ; s=\sum_{(k, n) \in F} c_{k n} \mathbf{T}_{\alpha k} \mathbf{M}_{\beta n} g, c_{k n} \in \mathbb{C}, \operatorname{card}(F) \leq N\right\}$

Proposition 3.3. Let $1 \leq p \leq q<\infty, \alpha>0$ and $g \in M_{1}$, let $\mathscr{D}:=\left\{\mathbf{T}_{\alpha k} \mathbf{M}_{\beta n} g ; k, n \in \mathbb{Z}\right\}$ be a dictionary given by a Gabor frame for $L_{2}(\mathbb{R})$, and for $\alpha=\left(\frac{1}{p}-\frac{1}{q}\right)+1$. Then we have:

If $s \in \Sigma_{N}(\mathscr{D}), \quad$ then $\quad\|s\|_{M_{p}} \leq C N^{\alpha}\|s\|_{M_{q}} \quad \forall N=1,2, \ldots$.

for some positive constant $C:=C(\alpha, \beta, g)$. 
Proof. Let $s \in \Sigma_{N}$ which has a Gabor expansion:

$s=\sum_{(k, n) \in F} c_{k n} \mathbf{T}_{\alpha k} \mathbf{M}_{\beta n} g$,

for some indexed set $F$ with $\operatorname{card}(F) \leq N$ and some coefficients $c_{k n}$, and for simplicity of notations we let $g_{k n}=\mathbf{T}_{\alpha k} \mathbf{M}_{\beta n} g$. Taking the $M_{p}$-norm for $s$ and using formula (2.12) we have:

$$
\begin{aligned}
\|s\|_{M_{p}}^{p} & =\left\|\mathscr{V}_{g} s\right\|_{L_{p}}^{p} \\
& \leq \int_{\mathbb{R}} \int_{\mathbb{R}}\left(\sum_{(k, n) \in F}\left|c_{k n} \mathscr{V}_{g} g_{k n}(x, y)\right|\right)^{p} d x d y
\end{aligned}
$$

and applying the Hölder's inequality for $\sum_{(k, n) \in F}\left|c_{k n} \mathscr{V}_{g} g_{k n}(x, y)\right|$, for $1 \leq p<\infty$, and using the fact that $q^{\prime} \leq p^{\prime}$ we get

$$
\begin{aligned}
\|s\|_{M_{p}} & \leq\left(\sum_{(k, n) \in F}\left|c_{k n}\right|^{p}\right)^{1 / p} \cdot\left(\int_{\mathbb{R}} \int_{\mathbb{R}}\left(\left[\sum_{(k, n) \in F}\left|\mathscr{V}_{g} g_{k n}(x, y)\right|^{q^{\prime}}\right]^{1 / q^{\prime}}\right)^{p} d x d y\right)^{1 / p} \\
& \leq N^{\frac{1}{p}}\left(\sup _{(k, n) \in F}\left|c_{k n}\right|\right) \cdot N^{\frac{1}{q^{\prime}}}\left(\int_{\mathbb{R}} \int_{\mathbb{R}}\left(\sup _{(k, n) \in F}\left|\mathscr{V}_{g} g_{k n}(x, y)\right|\right)^{p} d x d y\right)^{\frac{1}{p}} \\
& =N^{\frac{1}{p}-\frac{1}{q}+1} \cdot\left(\sup _{(k, n) \in F}\left|c_{k n}\right|\right) \cdot\left\|\mathscr{V}_{g} g_{k^{\prime} n^{\prime}}\right\|_{L_{p}}, \text { forsome }\left(k^{\prime}, n^{\prime}\right) \in F \\
& \leq N^{\frac{1}{p}-\frac{1}{q}+1} \cdot\left(\sup _{(k, n) \in F}\left|c_{k n}\right|\right) \cdot C^{\prime}\|g\|_{M_{p}}
\end{aligned}
$$

where we used part (2) of Theorem (2.3) in the last inequality. Now, since $g \in M_{1}$ and $1 \leq p<\infty$, then $\|g\|_{M_{p}} \leq\|g\|_{M_{1}}$ which is finite. Moreover, Since $s \in M_{\infty}$, Theorem (2.4) implies that there exist constants $A, B>0$ (depends only on $\alpha, \beta$ and $g$ ) such that for $p=q=\infty$ we have

$\|s\|_{M_{p}} \leq C^{\prime} \cdot N^{\frac{1}{p}-\frac{1}{q}+1}\left(B .\|s\|_{M_{\infty}}\right) \cdot\|g\|_{M_{1}}$

Furthermore, we know that $\|s\|_{M_{\infty}} \leq\|s\|_{M_{q}}$ for $1 \leq q<\infty$, hence

$\|s\|_{M_{p}} \leq \mathbf{C} N^{\frac{1}{p}-\frac{1}{q}+1}\|s\|_{M_{q}}$

for a constant $\mathbf{C}$ independent of $s$, which completes the proof.

Remark $3.4([10])$. . By the monotonicity of the sequence $\left(\sigma_{N}(f)_{M_{q}}\right)$, we have the following equivalence relation:

$$
\left(\sum_{N=1}^{\infty}\left[N^{\alpha} \sigma_{N}(f)_{M_{q}}\right]^{\lambda} \frac{1}{N}\right)^{1 / \lambda} \asymp\left(\sum_{N=0}^{\infty}\left[\left(2^{N}\right)^{\alpha} \sigma_{2^{N}}(f)_{M_{q}}\right]^{\lambda}\right)^{1 / \lambda}
$$

for each $\alpha>0$ and $0<\lambda<\infty$. Furthermore, this equivalence means that there exists finite constants $A_{1}, A_{2}>0$, such that

$$
\begin{aligned}
A_{1}\left(\sum_{N=1}^{\infty}\left[N^{\alpha} \sigma_{N}(f)_{M_{q}}\right]^{\lambda} \frac{1}{N}\right)^{1 / \lambda} & \leq\left(\sum_{N=0}^{\infty}\left[\left(2^{N}\right)^{\alpha} \sigma_{2^{N}}(f)_{M_{q}}\right]^{\lambda}\right)^{1 / \lambda} \\
& \leq A_{2}\left(\sum_{N=1}^{\infty}\left[N^{\alpha} \sigma_{N}(f)_{M_{q}}\right]^{\lambda} \frac{1}{N}\right)^{1 / \lambda}
\end{aligned}
$$


Theorem 3.5. Let $\left\{\mathbf{T}_{\alpha k} \mathbf{M}_{\beta n} g ; k, n \in \mathbb{Z}\right\}$ be a Gabor frame for $L_{2}(\mathbb{R}), \Sigma_{N}$ as defined in (3.3), $1 \leq p \leq$ $q<\infty$, and $\alpha=\frac{1}{p}-\frac{1}{q}+1$. Then we have

if $\quad \sum_{N=1}^{\infty}\left(N^{\alpha} \sigma_{N}(f)_{M_{q}}\right)^{p} \frac{1}{N}<\infty \quad$ then $\quad f \in M_{p}$

Proof. Given a function $f$ belongs to the modulation space $M_{\infty}$ and has a Gabor expansion

$f=\sum_{k, n \in \mathbb{Z}} \lambda_{k n} \mathbf{T}_{\alpha k} \mathbf{M}_{\beta n} g$

for some window function $g \in M_{1}$. Moreover, suppose that the approximation error of approximating $f$ by elements from $\Sigma_{N}$ is measured in an $M_{q}$-norm and satisfies

$$
\left(\sum_{N=1}^{\infty}\left[N^{\alpha} \sigma_{N}(f)_{M_{q}}\right]^{p} \frac{1}{N}\right)<\infty
$$

for any $\alpha>0$, and $1 \leq p<\infty$, we need to show that $\|f\|_{M_{p}}<\infty$.

For all $N \in \mathbb{N}$, let $s_{N} \in \Sigma_{2^{N}}$ be a near-best approximant to $f$ from $\Sigma_{2^{N}}$, i.e.,

$\left\|f-s_{N}\right\|_{M_{q}} \equiv \sigma_{2^{N}}(f)_{M_{q}}$

Furthermore, we can assume that every $f$ in $M_{\infty}$ can be written as

$f=\lim _{k \rightarrow \infty} \sum_{N=1}^{k}\left(s_{N}-s_{N-1}\right)=\sum_{N=1}^{\infty}\left(s_{N}-s_{N-1}\right)$

where $s_{0}=0$. Now, taking the $M_{p}$-norm for $f$ we get

$\|f\|_{M_{p}} \leq \sum_{N=1}^{\infty}\left\|s_{N}-s_{N-1}\right\|_{M_{p}}$

Since $\Sigma_{2^{N-1}} \subseteq \Sigma_{2^{N}}$. Then $\left(s_{N}-s_{N-1}\right) \in \Sigma_{2^{N}}$. So we can apply our result in Proposition (3.3) for $\left(s_{N}-s_{N-1}\right)$ as follows: There exist $C>0$ such that

$\left\|s_{N}-s_{N-1}\right\|_{M_{p}} \leq C\left(2^{N}\right)^{\alpha}\left\|s_{N}-s_{N-1}\right\|_{M_{q}}$

for the given $\alpha>0$.

Moreover, from assumption (3.9) and from the monotonicity of $\left(\sigma_{N}(f)_{M_{q}}\right)$, we have

$\left\|s_{N}-s_{N-1}\right\|_{M_{q}} \leq C^{\prime} \sigma_{2^{N-1}}(f)_{M_{q}}$, for some constant $C^{\prime}>0$.

Back to Equation (3.10), using the previous inequality, we have,

$\|f\|_{M_{p}} \leq C_{2}^{\prime} \sum_{N=0}^{\infty}\left[\left(2^{N}\right)^{\alpha} \sigma_{2^{N}}(f)_{M_{q}}\right]$

Using Remark 3.4 for $\lambda=1$ and our assumption in (3.8) above we get

$$
\|f\|_{M_{p}} \leq C_{2}^{\prime \prime}\left(\sum_{N=1}^{\infty}\left[N^{\alpha} \sigma_{N}(f)_{M_{q}}\right] \frac{1}{N}\right)<\infty
$$

Thus, our proof is complete and we have $\|f\|_{M_{p}}<\infty$, i.e., $f \in M_{p}$. 


\section{Characterization of Modulation Spaces $M_{p, q}$}

Now it is natural according to the later results that one thinking in the characterization of the modulation spaces $M_{p, q}$ using the Gabor frames and nonlinear approximation, and if it is possible to characterize an arbitrary function $f$ belonging to some modulation space like $M_{\infty}$ using elements from the same space, knowing that its approximation error measured in the $M_{p, q}$-norm satisfying some condition.

Let $\mathscr{D}:=\left\{\mathbf{T}_{\alpha k} \mathbf{M}_{\beta n} g ; k, n \in \mathbb{Z}\right\}$ be a dictionary given by a Gabor frame for $L_{2}(\mathbb{R})$, and define for all $N \in \mathbb{N}:$

$\Sigma_{N}(\mathscr{D})=\left\{s \in M_{\infty} ; s=\sum_{(k, n) \in F} c_{k n} \mathbf{T}_{\alpha k} \mathbf{M}_{\beta n} g, c_{k n} \in \mathbb{C}, \operatorname{card}(F) \leq N\right\}$

$\sigma_{N}(f)_{M_{p, q}}=\inf _{s \in \Sigma_{N}}\|f-s\|_{M_{p, q}}$

We can solve this problem in one direction. More precisely, for $\Sigma_{N}$ defined in Equation (4.1), we have

Theorem 4.1. Let $\left\{\mathbf{T}_{\alpha k} \mathbf{M}_{\beta n} g ; k, n \in \mathbb{Z}\right\}$ be a Gabor frame for $L_{2}(\mathbb{R}), \Sigma_{N}$ as defined in Equation (4.1), $1 \leq p_{1} \leq p<\infty, 1 \leq q_{1} \leq q<\infty$, and $\alpha=\left(\frac{1}{p_{1}}+\frac{1}{q_{1}}\right)-\left(\frac{1}{p}+\frac{1}{q}\right)+2$. Then, for some $\tau>0$, we have:

if $\quad \sum_{N=1}^{\infty}\left(N^{\alpha} \sigma_{N}(f)_{M_{p, q}}\right)^{\tau} \frac{1}{N}<\infty, \quad$ then $f \in M_{p_{1}, q_{1}}$

Before starting the proof of the theorem, we must show that the Bernstein estimate theorem can be applied to these modulation spaces under our new definition of $\Sigma_{N}$ and a given rate $\alpha>0$.

Proposition 4.2. (Bernstein Inequality and Modulation Spaces $M_{p, q}$ ).

Let $1 \leq p_{1} \leq p<\infty, 1 \leq q_{1} \leq q<\infty$ and $\alpha>0$. Let $g \in M_{1}$, and $\mathscr{D}:=\left\{\mathbf{T}_{\alpha k} \mathbf{M}_{\beta n} g ; k, n \in \mathbb{Z}\right\}$ be a dictionary given by a Gabor frame for $L_{2}(\mathbb{R})$. Then for $\alpha=\left(\frac{1}{p_{1}}+\frac{1}{q_{1}}\right)-\left(\frac{1}{p}+\frac{1}{q}\right)+2$, we have:

if $s \in \Sigma_{N}(\mathscr{D}), \quad$ then $\quad\|s\|_{M_{p_{1}, q_{1}}} \leq C N^{\alpha}\|s\|_{M_{p, q}}, \quad \forall N=1,2, \ldots$

for some positive constant $C:=C(\alpha, \beta, g)$.

Proof. Let $s \in \Sigma_{N}$. Then

$s=\sum_{(k, n) \in F} c_{k n} \mathbf{T}_{\alpha k} \mathbf{M}_{\beta n} g=\sum_{k} \sum_{n} c_{k n} \mathbf{T}_{\alpha k} \mathbf{M}_{\beta n} g$

for some indexed set $F$ with $\operatorname{card}(F) \leq N$ and some coefficients $c_{k n}$. For simplicity of notations we let $g_{k n}=\mathbf{T}_{\alpha k} \mathbf{M}_{\beta n} g$. Now, taking the $M_{p_{1}, q_{1}}$-norm for $s$ and using formula (2.12) we get

$$
\begin{aligned}
\|s\|_{M_{p_{1}, q_{1}}}^{q_{1}} & =\left\|\mathscr{V}_{g} s\right\|_{L_{p_{1}, q_{1}}}^{q_{1}} \\
& \leq \int_{\mathbb{R}}\left(\int_{\mathbb{R}}\left[\sum_{k} \sum_{n}\left|c_{k n} \| \mathscr{V}_{g} g_{k n}(x, y)\right|\right]^{p_{1}} d x\right)^{q_{1} / p_{1}} d y
\end{aligned}
$$

Now, keeping in mind that we are working over a finite indexed set $F$, using the Hölder's inequality over the internal index, and $\frac{1}{p_{1}}+\frac{1}{p_{1}^{\prime}}=1$, we have for each $x, y$

$\sum_{k}\left(\sum_{n}\left|c_{k n}\right|\left|\mathscr{V}_{g} g_{k n}(x, y)\right|\right) \leq \sum_{k}\left(\left(\sum_{n}\left|c_{k n}\right|^{p_{1}}\right)^{\frac{1}{p_{1}}} \cdot\left(\sum_{n}\left|\mathscr{V}_{g} g_{k n}(x, y)\right|^{p_{1}^{\prime}}\right)^{\frac{1}{p_{1}^{\prime}}}\right)$

Again, using the Hölder's inequality over the external index, and $\frac{1}{q_{1}}+\frac{1}{q_{1}^{\prime}}=1$, the right hand side of the last inequality will be less than or equal to

$$
\left(\sum_{k}\left[\sum_{n}\left|c_{k n}\right|^{p_{1}}\right]^{\frac{q_{1}}{p_{1}}}\right)^{\frac{1}{q_{1}}} \cdot\left(\sum_{k}\left[\sum_{n}\left|\mathscr{V}_{g} g_{k n}(x, y)\right|^{p_{1}^{\prime}}\right]^{\frac{q_{1}^{\prime}}{p_{1}^{\prime}}}\right)^{\frac{1}{q_{1}^{\prime}}}
$$


Now, simplifying the first part of the expression in (4.6), we get

$$
\left(\sum_{k}\left[\sum_{n}\left|c_{k n}\right|^{p_{1}}\right]^{\frac{q_{1}}{p_{1}}}\right)^{\frac{1}{q_{1}}} \leq N^{\frac{1}{p_{1}}+\frac{1}{q_{1}}} \cdot\left(\sup _{(k, n) \in F}\left|c_{k n}\right|\right)
$$

But since $p_{1} \leq p$, then $p^{\prime} \leq p_{1}^{\prime}$, so

$$
\left[\sum_{n}\left|\mathscr{V}_{g} g_{k n}(x, y)\right|^{p_{1}^{\prime}}\right]^{1 / p_{1}^{\prime}} \leq\left[\sum_{n}\left|\mathscr{V}_{g} g_{k n}(x, y)\right|^{p^{\prime}}\right]^{1 / p^{\prime}}
$$

and we get

$$
\begin{aligned}
\left(\sum_{k}\left[\sum_{n}\left|\mathscr{V}_{g} g_{k n}(x, y)\right|^{p_{1}^{\prime}}\right]^{\frac{q_{1}^{\prime}}{p_{1}^{\prime}}}\right)^{\frac{1}{q_{1}^{\prime}}} & \leq\left(\sum_{k}\left[N^{\frac{1}{p^{\prime}}} \sup _{n}\left|\mathscr{V}_{g} g_{k n}(x, y)\right|\right]^{q_{1}^{\prime}}\right)^{\frac{1}{q_{1}^{\prime}}} \\
& \leq N^{\frac{1}{p^{\prime}}}\left(\sum_{k}\left|\mathscr{V}_{g} g_{k n^{\prime}}(x, y)\right|^{q_{1}^{\prime}}\right)^{\frac{1}{q_{1}^{\prime}}}
\end{aligned}
$$

Since $q_{1} \leq q$, we must have $q^{\prime} \leq q_{1}^{\prime}$. Hence we get

$$
\begin{aligned}
\left(\sum_{k}\left|\mathscr{V}_{g} g_{k n^{\prime}}(x, y)\right|^{q_{1}^{\prime}}\right)^{1 / q_{1}^{\prime}} & \leq\left(\sum_{k}\left|\mathscr{V}_{g} g_{k n^{\prime}}(x, y)\right|^{q^{\prime}}\right)^{1 / q^{\prime}} \\
& =N^{\frac{1}{q^{\prime}}}\left|\mathscr{V}_{g} g_{k^{\prime} n^{\prime}}(x, y)\right|
\end{aligned}
$$

Therefore

$$
\left(\sum_{k}\left[\sum_{n}\left|\mathscr{V}_{g} g_{k n}(x, y)\right|^{p_{1}^{\prime}}\right]^{\frac{q_{1}^{\prime}}{p_{1}^{\prime}}}\right)^{\frac{1}{q_{1}^{\prime}}} \leq N^{2-\left(\frac{1}{p}+\frac{1}{q}\right)}\left|\mathscr{V}_{g} g_{k^{\prime} n^{\prime}}(x, y)\right|
$$

Combining our results we get

$$
\begin{aligned}
\|s\|_{M_{p_{1}, q_{1}} \leq} \leq & \left(\int_{\mathbb{R}}\left(\int_{\mathbb{R}}\left[\sum_{k} \sum_{n}\left|c_{k n}\right|\left|\mathscr{V}_{g} g_{k n}(x, y)\right|\right]^{p_{1}} d x\right)^{q_{1} / p_{1}} d y\right)^{\frac{1}{q_{1}}} \\
& \left.\left.\left.\left(\sum_{k}\left[\sum_{n}\left|\mathscr{V}_{g} g_{k n}(x, y)\right|^{p_{1}^{\prime}}\right]^{\frac{q_{1}^{\prime}}{p_{1}^{\prime}}}\right]^{\frac{1}{q_{1}^{\prime}}}\right]^{p_{1}} d x\right)^{q_{1} / p_{1}} d y\right)^{\frac{1}{q_{1}}} \\
\leq & N^{\left(\frac{1}{p_{1}}+\frac{1}{q_{1}}\right)-\left(\frac{1}{p}+\frac{1}{q}\right)+2} \cdot \sup _{(k, n) \in F}\left|c_{k n}\right| \cdot\left(\int_{\mathbb{R}}\left(\int_{\mathbb{R}}\left|\mathscr{V}_{g} g_{k^{\prime} n^{\prime}}(x, y)\right|^{p_{1}} d x\right)^{q_{1} / p_{1}} d y\right)^{1 / q_{1}}
\end{aligned}
$$

Using Equation (2.12) and Theorem (2.4) for $p, q<\infty$ we have

$$
\begin{aligned}
\|s\|_{M_{p_{1}, q_{1}}} & \leq N^{\left(\frac{1}{p_{1}}+\frac{1}{q_{1}}\right)-\left(\frac{1}{p}+\frac{1}{q}\right)+2} \cdot\|s\|_{M_{\infty} \cdot\left\|\mathscr{V}_{g} g_{k^{\prime} n^{\prime}}\right\|_{L_{p_{1}, q_{1}}}} \\
& \leq C N^{\left(\frac{1}{p_{1}}+\frac{1}{q_{1}}\right)-\left(\frac{1}{p}+\frac{1}{q}\right)+2} \cdot\|s\|_{M_{p, q} \cdot\left\|g_{k^{\prime} n^{\prime}}\right\|_{M_{p_{1}, q_{1}}}}
\end{aligned}
$$

for some positive constant $C:=C(\alpha, \beta, g)$. Moreover, using part (2) of Theorem (2.3) and our assumption that $g \in M_{1}$ and $1 \leq p_{1}, q_{1}<\infty$ we get our result. 


\section{Proof of Theorem (4.1).}

Given a function $f$ belongs to the modulation space $M_{\infty}$ and has a Gabor expansion

$f=\sum_{k, n \in \mathbb{Z}} \lambda_{k n} \mathbf{T}_{\alpha k} \mathbf{M}_{\beta n} g$

for some window function $g \in M_{1}$. Suppose that the approximation error of approximating $f$ by elements from $\Sigma_{N}$ is measured in an $M_{p, q}$-norm and satisfies

$$
\left(\sum_{N=1}^{\infty}\left[N^{\alpha} \sigma_{N}(f)_{M_{p, q}}\right]^{\tau} \frac{1}{N}\right)<\infty, \text { for some } \tau>0
$$

we need to show that $\|f\|_{M_{p_{1}, q_{1}}}<\infty$.

Let $s_{N} \in \Sigma_{2^{N}}$ be a near-best approximant to $f$ from $\Sigma_{2^{N}}$, for all $N \in \mathbb{N}$, i.e.,

$$
\left\|f-s_{N}\right\|_{M_{p, q}} \equiv \sigma_{2^{N}}(f)_{M_{p, q}}
$$

Since every $f$ in $M_{\infty}$ can be written as

$f=\sum_{N=1}^{\infty}\left(s_{N}-s_{N-1}\right)$

where $s_{0}=0$. Taking the $M_{p_{1}, q_{1}}$-norm for $f$ we get

$\|f\|_{M_{p_{1}, q_{1}}} \leq \sum_{N=1}^{\infty}\left\|s_{N}-s_{N-1}\right\|_{M_{p_{1}, q_{1}}}$

Since $\left(s_{N}-s_{N-1}\right) \in \Sigma_{2^{N}}$. So, from Proposition (4.2), there exist $C>0$ such that

$\left\|s_{N}-s_{N-1}\right\|_{M_{p_{1}, q_{1}}} \leq C\left(2^{N}\right)^{\alpha}\left\|s_{N}-s_{N-1}\right\|_{M_{p, q}}$

for the given $\alpha$. Moreover, from assumption (4.8) and from the monotonicity of $\left(\sigma_{N}(f)_{M_{p, q}}\right)$, we have

$$
\left\|s_{N}-s_{N-1}\right\|_{M_{p, q}} \leq C^{\prime} \sigma_{2^{N-1}}(f)_{M_{p, q}}, \text { for some constant } C^{\prime}>0 .
$$

Back to Equation (4.9), and using the previous inequality we have,

$\|f\|_{M_{p_{1}, q_{1}}} \leq C_{2}^{\prime} \sum_{N=0}^{\infty}\left[\left(2^{N}\right)^{\alpha} \sigma_{2^{N}}(f)_{M_{p, q}}\right]$

and using Remark 3.4 for $\lambda=1$ and our assumption in (4.7) above we get

$$
\|f\|_{M_{p_{1}, q_{1}}} \leq C_{2}^{\prime \prime}\left(\sum_{N=1}^{\infty}\left[N^{\alpha} \sigma_{N}(f)_{M_{p, q}}\right] \frac{1}{N}\right)<\infty
$$

thus, we have $\|f\|_{M_{p_{1}, q_{1}}}<\infty$, and $f \in M_{p_{1}, q_{1}}$.

\section{Competing Interests}

The authors declare no competing interests. 


\section{References}

[1] Stechkin S. B. On Absolute Convergence of Orthogonal Series, Dok. Akad. Nauk SSSR, 102, 37-40 (1955).

[2] Ronald D, Temlyakov V. N. Some Remarks on Greedy Algoritheorems, Advances in Comp. Math., 5(2-3), 173-187 (1996).

[3] Feichtinger H. Modulation Spaces on Locally Compact Abelian Groups. Technical Report, Unversity of Vienna, 1983.

[4] Feichtinger H. Atomic Characterization of Modulation Spaces Through Gabor-Type Representations. Proc. Conference on Constructive Function Theory. Edmonton, Rocky Mountain J. Math., 19, 113-126 (1986).

[5] Feichtinger H, Gröchenig K. Gabor Wavelets and the Heisenberg Group: Gabor Expansions and Short Time Fourier Transform from the Group Theoretical Point of View. In Charles K. Chui. editor, Wavelets: A Tutorial in Theory and Applications, Academic Press. Boston, 359-398 (1992).

[6] Gröchenig K. Foundations of Time-Frequency Analysis, Applied and Numerical Harmonic Analysis, Birkhäuser. Boston, 2000.

[7] Feichtinger H, Gröchenig K, Walnut D. Wilson Bases and Modulation Spaces, Math. Nacher, 155, 7-17 (1992).

[8] Gröchenig K, Samarah S. Nonlinear Approximation with Local Fourier basis, Constructive Approximation, 6(3), 317-341 (2000).

[9] Samarah S. Approximation Theory and Modulation Spaces. PhD thesis. Univ. of Connecticut, 1998.

[10] Ronald D. Nonlinear Approximation, Acta Numerica, Cambridge Univ. Press. Cambridge, 51-150 (1998). 